\title{
Antitumor Effect of KX-01 through Inhibiting Src Family Kinases and Mitosis
}

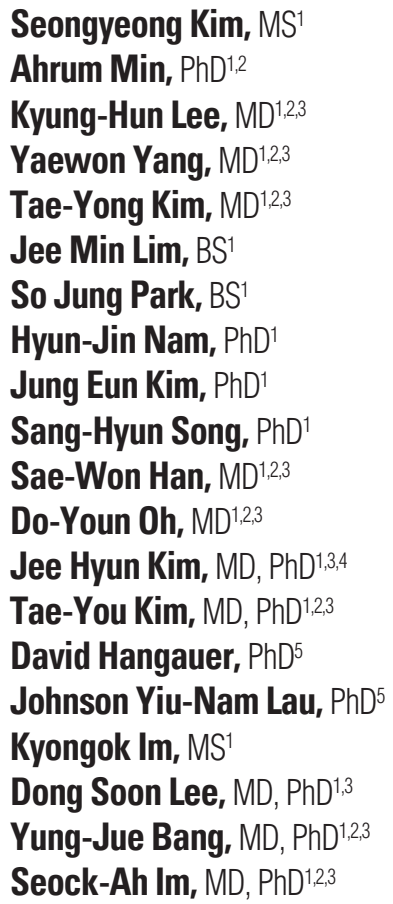

${ }^{1}$ Cancer Research Institute, Seoul National University, Seoul, ${ }^{2}$ Biomedical Research Institute, Seoul National University Hospital, Seoul, ${ }^{3}$ Department of Internal Medicine, Seoul National University College of Medicine, Seoul, ${ }^{4}$ Department of Internal Medicine, Seoul National University Bundang Hospital, Seoul National University College of Medicine, Seongnam, Korea, ${ }^{5}$ Kinex Pharmaceutical Corporation, New York State Center of Excellence in Bioinformartics and Life Sciences, NY, USA

Correspondence: Seock-Ah Im, MD, PhD Department of Internal Medicine, Seoul National University College of Medicine, 101 Daehak-ro, Jongno-gu, Seoul 03080, Korea

Tel: 82-2-2072-0850

Fax: 82-2-762-9662

E-mail: moisa@snu.ac.kr

Received April 21, 2016

Accepted September 17, 2016

Published Online October 6, 2016

\section{Purpose}

KX-01 is a novel dual inhibitor of Src and tubulin. Unlike previous Src inhibitors that failed to show clinical benefit during treatment of breast cancer, KX-01 can potentially overcome the therapeutic limitations of current Src inhibitors through inhibition of both Src and tubulin. The present study further evaluates the activity and mechanism of KX-01 in vitro and in vivo.

\section{Materials and Methods}

The antitumor effect of KX-01 in triple negative breast cancer (TNBC) cell lines was determined by MTT assay. Wound healing and immunofluorescence assays were performed to evaluate the action mechanisms of KX-01. Changes in the cell cycle and molecular changes induced by KX-01 were also evaluated. A MDA-MB-231 mouse xenograft model was used to demonstrate the in vivo effects.

\section{Results}

KX-01 effectively inhibited the growth of breast cancer cell lines. The expression of phospho-Src and proliferative-signaling molecules were down-regulated in KX-01-sensitive TNBC cell lines. In addition, migration inhibition was observed by wound healing assay. KX-01induced G2/M cell cycle arrest and increased the aneuploid cell population in KX-01-sensitive cell lines. Multi-nucleated cells were significantly increased after KX-01 treatment. Furthermore, KX-01 effectively delayed tumor growth in a MDA-MB-231 mouse xenograft model.

\section{Conclusion}

KX-01 effectively inhibited cell growth and migration of TNBC cells. Moreover, this study demonstrated that KX-01 showed antitumor effects through the inhibition of Src signaling and the induction of mitotic catastrophe. The antitumor effects of KX-01 were also demonstrated in vivo using a mouse xenograft model.

\section{Key words}

Src kinase inhibitor, Mitotic catastrophe, Microtubules,

KX-01, Triple negative breast neoplasms 


\section{Introduction}

Breast cancer is classified into several subtypes based on the expression patterns of estrogen receptor (ER), progesterone receptor, and human epidermal growth factor receptor type 2 (HER2) [1]. When all three receptors are absent, they are classified as triple negative breast cancer (TNBC). This type of cancer is normally associated with a very poor prognosis because, unlike other subtypes, no known therapeutic agents can specifically target TNBC [1]. Patients with this disease have been treated with cytotoxic chemotherapy alone; however, chemo-resistance develops easily, resulting in treatment failure. Therefore, new therapeutic strategies for TNBC are urgently needed.

One of the reasonable targets of treatment is the Src family kinases (SFKs). SFKs are non-receptor tyrosine kinases that are activated in various solid tumors, including breast cancer [2-5]. Activation of Src signaling can induce cell proliferation, invasion, and metastasis, which are hallmarks of aggressive tumors; therefore, Src has been an attractive therapeutic target, especially for TNBC [4]. A previous study also confirmed that cytoplasmic Src expression was much higher in TNBC patient samples than non-TNBC patient samples [2]. Although several Src inhibitors have been developed, none have produced remarkable responses as mono-therapeutic agents [6-8]. Therefore, a new Src inhibitor is needed for TNBC treatment.

Microtubule dynamics are precisely regulated for accurate cell division during mitosis. Several drugs target microtubule dynamics, and their application can lead to mitotic catastrophe in cancer cells. Mitotic catastrophe is a type of cell death induced by either aberrant mitosis or the accumulation of damaged chromosomes [9-13]. Tumor cell populations are usually composed of high numbers of tetraploid cells that are more prone to mitotic aberrations and more sensitive to mitotic catastrophe-inducing agent [13]. Moreover, mitotic catastrophe can be exploited to eliminate apoptosis-resistant cancer cells [11]. Therefore, the induction of mitotic catastrophe may be an alternative method of overcoming chemoresistance caused by apoptosis-resistant cells.

KX-01 is a novel, non-ATP-competitive Src inhibitor that also inhibits tubulin polymerization [14-18]. KX-01 has shown activity against various types of cancers, including TNBC, ER-positive breast cancer, and mucinous ovarian cancer, both in vitro and in vivo $[14,15,18]$. However, previous studies focused more on verifying the Src signaling inhibitory effects of KX-01 and only showed decreasing phosphorylated Src (p-Src) level in vivo. Moreover, the microtubule polymerization inhibitory effects of KX-01 have not been studied in depth. The present study evaluated the activity of KX-01 against a broad range of TNBC cell lines and investigated the mechanism of action in greater depth. We focused on verifying that KX-01 actually inhibits its primary target, Src, and confirmed it down-regulates the expression of p-Src in vitro. Moreover, we showed that the tubulin inhibitory effect of KX-01 causes induction of mitotic catastrophe. We also evaluated the overall effect of KX-01 in an established mouse model. Our results provide further insight into the mechanistic activity of KX-01, which may guide the development of better therapeutic strategies based on KX-01 for the treatment of breast cancer.

\section{Materials and Methods}

\section{Antibodies and reagents}

KX-01 was kindly provided by Kinex Pharmaceutical (Buffalo, NY). The compound was initially dissolved in dimethyl sulfoxide (DMSO) and stored at $-80^{\circ} \mathrm{C}$. Paclitaxel was obtained from Samyang Co., Ltd. (Seoul, Korea). Antibodies against phosphorylated (p)-Src (Y416), FAK, p-p130cas, p-ERK (T202/Y204), ERK (p44/p42), p-AKT (S473), AKT, p-STAT3 (Y705), and STAT3 were purchased from Cell Signaling Technology (Danvers, MA), p-FAK (Y397) and p130cas were obtained from BD Biosciences (San Jose, CA), actin was acquired from Sigma Aldrich (St. Louis, MO), and Src and p-FAK (Y861) was obtained from Santa Cruz Biotechnology (Santa Cruz, CA).

\section{Cell lines and cell culture}

Human breast cancer cell lines (BT-474, BT-549, HCC1937, Hs578T, MCF7, MDA-MB-231, MDA-MB-468, SK-BR-3, and T47D) verified using short tandem repeat analysis were purchased from the American Type Culture Collection (ATCC; Manassas, VA). The cells were cultured in RPMI-1640 medium (Thermo Fisher Scientific Inc., Waltham, MA) supplemented with $10 \%$ fetal bovine serum (Life Technologies, Carlsbad, CA) and $10 \mu \mathrm{g} / \mathrm{mL}$ gentamicin (Cellgro, Manassas, VA) at $37^{\circ} \mathrm{C}$ under $5 \% \mathrm{CO}_{2}$.

\section{Cell growth inhibitory assay}

Cells were seeded in 96-well plates and incubated overnight at $37^{\circ} \mathrm{C}$ under $5 \% \mathrm{CO}_{2}$. The cells were exposed to increasing concentrations of $\mathrm{KX}-01$ or paclitaxel for 3 days. After drug treatment, $50 \mu \mathrm{L}$ of 3-(4,5-dimethylthiazol-2-yl)2,5-diphenyltetrazolim bromide solution (Sigma Aldrich) was added to each well and the plates were incubated for 4 hours at $37^{\circ} \mathrm{C}$. After dissolving the formazan crystals with 
$150 \mu \mathrm{L}$ of DMSO, the absorbance of each well was measured at $540 \mathrm{~nm}$ using a VersaMax microplate reader (Molecular Devices, Sunnyvale, CA). The absorbance and $\mathrm{IC}_{50}$ of KX-01 were analyzed using the SigmaPlot software (SPSS Inc., Chicago, IL). Six replicates were included in each dose and at least three independent experiments were conducted.

\section{Western blot analysis}

The Western blot analytic method was previously reported [19]. Proteins were extracted, after which equal amount were separated by sodium dodecyl sulfate polyacrylamide gel electrophoresis and transferred to nitrocellulose membranes. After blocking with buffer, the blots were incubated with primary antibodies, followed by horseradish peroxidase-conjugated anti-rabbit or anti-mouse secondary antibodies. Antibody binding was detected using an enhanced chemiluminescence system according to the manufacturer's protocol (Amersham Biosciences, Piscataway, NJ).

\section{Wound healing assay}

Cells were seeded in 6-well plates and incubated overnight at $37^{\circ} \mathrm{C}$ under $5 \% \quad \mathrm{CO}_{2}$. The cell monolayer was then scratched with a pipette tip and the cells were incubated with medium alone or containing $20 \mathrm{nmol} / \mathrm{L} \mathrm{KX-01}$. After 48 hours, the plates were examined by light microscopy (Olympus, Tokyo, Japan) to monitor restoration of the cell monolayer. The percentage of the filled gap was analyzed using the SigmaPlot software.

\section{Cell cycle analysis}

KX-01 treated cells were harvested with trypsin, fixed with cold $70 \%$ ethanol, and stored at $-20^{\circ} \mathrm{C}$ for at least 24 hours. The cells were then washed in phosphate buffered saline (PBS) and incubated with $10 \mu \mathrm{g} / \mathrm{mL}$ RNase A (Sigma Aldrich) at $37^{\circ} \mathrm{C}$ for 20 minutes. Next, the cells were stained with $20 \mu \mathrm{g} / \mathrm{mL}$ propidium iodide (Sigma Aldrich), after which the DNA contents were quantified using a FACS Calibur flow cytometer (BD Biosciences).

\section{Immunofluorescence assay}

This experiment was conducted following a previously reported method [20]. Briefly, cells were plated on coverslips and incubated with KX-01 for 24 hours or 48 hours. The primary antibody ratio was 1:50 to 1:200. Immunofluorescence was visualized using a Zeiss LSM 510 laser scanning microscope (Zeiss, Jena, Germany).

\section{In vivo studies}

All animal experiments were carried out at the animal facility of Seoul National University (Seoul, Korea) in accordance with institutional guidelines. To measure the in vivo activity of KX-01, 5-week-old female BALB / c athymic nude mice were purchased from Central Lab Animal, Inc. (Seoul, Korea). The mice were allowed to acclimatize for 1 week before receiving a subcutaneous injection of MDA-MB-231 cancer cells $\left(5.0 \times 10^{7}\right)$ in $200 \mu \mathrm{L}$ of PBS. When tumors reached a volume of $150 \mathrm{~mm}^{3}$, the mice were randomly divided into two groups, a control group that received vehicle $(10 \%$ 2-hydroyl-propyl- $\beta$-cyclodextrine [Sigma Aldrich] diluted in PBS solution), and a treatment group that received $5 \mathrm{mg} / \mathrm{kg}$ KX-01 in vehicle solution twice daily for 4 weeks. The vehicle solution and KX-01 were administered orally. The tumor was measured every other day using calipers and the volume was calculated with the following formula: [(width $)^{2} \times($ height $\left.)\right]$ /2. At the end of the measurement period, the mice were euthanized with $\mathrm{CO}_{2}$. The tumors were then excised and fixed in neutral-buffered formalin for routine histological examination and immunohistochemical staining. Total proteins were extracted from fresh tissue samples to assess the protein expression and Src activity.

\section{Immunohistochemistry}

Sections from individual paraffin-embedded xenograft tumor tissues were deparaffinized and rehydrated. Immunohistochemical detection of proliferating cells was determined using an anti-Ki-67 antibody (GeneTex, Irvine, CA). A terminal deoxynucletidyltransferase-mediated dUTP nick end labeling (TUNEL) assay was performed to detect apoptosis using an ApopTagIn Situ Apoptosis Detection Kit (Chemicon International, Temecula, $\mathrm{CA}$ ) according to the manufacturer's protocol.

\section{Statistical analysis}

Statistical analyses were conducted using SigmaPlot ver. 9.0. A two-sided Student's $t$ test was performed when appropriate. Results are expressed as the mean \pm standard deviations or standard errors. A p-value of $<0.1$ was considered statistically significant. All experiments were conducted in duplicate or triplicate and repeated at least twice. 


\section{Results}

\section{KX-01 effectively inhibits the growth of breast cancer cells and regulates SFK phosphorylation}

To verify the growth inhibitory effects of KX-01 on breast cancer cells, nine breast cancer cell lines were treated with KX-01 in vitro, and their inhibitory effects were evaluated using an MTT assay (data not shown). The luminal ER+ cell lines MCF7 and T47D, the HER2+ cell line SK-BR-3, and the TNBC cell lines MDA-MB-231, MDA-MB-468, and BT-549 were sensitive to $\mathrm{KX}-01$ with $\mathrm{IC}_{50}$ values lower than 0.1 $\mu \mathrm{mol} / \mathrm{L}$ (Table 1), whereas TNBC cell lines Hs578T and HCC1937 were resistant to KX-01. Four TNBC cell lines (MDA-MB-231, MDA-MB-468, BT-549, and Hs578T) were selected for further in vitro studies.

To determine if KX-01 directly inhibits the activity of Src and FAK, Western blotting was performed to measure the levels of total and phosphorylated proteins after treatment. p-Src in BT-549 significantly decreased following exposure to KX-01 (Fig. 1A). Moreover, FAK and p130cas phosphorylation, which are known to be regulated by Src, also decreased. Other sensitive cell lines, MDA-MB-231 and MDA-MB-468, showed similar responses (Fig. 1A, S1 Fig.). Therefore, these results indicate that KX-01 indeed inhibits Src activity and its downstream proteins in vitro.

Src signaling regulates invasion and metastasis [3]; therefore, we investigated whether the inhibition of Src signaling would lead to inhibited cell migration and invasion. Consistent with the Western blot results and a previous study using MDA-MB-231 cells [14], the wound healing assay showed that cell migration was inhibited by treatment in KX-01-sensitive BT-549 cells (Fig. 1B). In comparison, Hs578T cells, which were less sensitive to KX-01, showed no difference between the control and KX-01 treatment at $20 \mathrm{nmol} / \mathrm{L}$ con- centration. This concentration is known to only inhibit Src signaling, so these results infer that cell migration was inhibited by KX-01-sensitive cell lines because Src phosphorylation was suppressed.

To further explore the action mechanism of KX-01, we measured additional downstream signaling molecular-level changes after 24-hour KX-01 treatment. Src regulates survival and proliferation. Interestingly, while the $\mathrm{p}$-Src recovered 24 hours after the treatment of KX-01, regardless of the sensitivity (data not shown), only the sensitive cell lines had low p-AKT, p-ERK, and p-STAT3 (Fig. 1C).

These data indicate that KX-01 has effective antitumor effects against a broad range of TNBC cell lines, and these effects are related, at least in part, to inhibition of Src signaling by KX-01 treatment. Moreover, inhibition of Src by KX-01 not only inhibits cell migration, but also cell proliferation and survival signaling molecules.

\section{KX-01 treatment leads to G2/M arrest}

The cell cycle of four cell lines was analyzed using flow cytometry. Other reported Src inhibitors in previous studies were found to cause G1 cell cycle arrest [21]. However, unlike these reported Src inhibitors, we were able to demonstrate that KX-01 caused cells to arrest at the G2/ M cell phase in a dose-dependent manner (Fig. 2). TNBC cells sensitive to KX-01 displayed 2- to 4-fold increased G2/ M cell phase population, while G1 and S cell phases decreased. Conversely, the KX-01-insensitive cell, Hs578T, did not show increased G2/M cell phase populations in response to a $100 \mathrm{nmol} / \mathrm{L}$ concentration of $\mathrm{KX}-01$.

It is worth noting that G2/M cell cycle arrest was most prominent at $100 \mathrm{nM} \mathrm{KX-01,} \mathrm{suggesting} \mathrm{that} 100 \mathrm{nM}$ is more effective at inhibiting microtubule polymerization than 50 $\mathrm{nM}$.

Table 1. Growth inhibitory effect of KX-01

\begin{tabular}{|c|c|c|}
\hline Cell line & Subtype & $\mathrm{KX}-01 \mathrm{IC}_{50}($ mean $\pm \mathrm{SD}, \mu \mathrm{mol} / \mathrm{L})$ \\
\hline MCF7 & Luminal (ER+) & $0.0418 \pm 0.0010$ \\
\hline T47D & Luminal (ER+/PR+) & $0.0435 \pm 0.0423$ \\
\hline BT-474 & HER2 & $0.1286 \pm 0.0076$ \\
\hline SK-BR-3 & HER2 & $0.0338 \pm 0.0010$ \\
\hline BT-549 & Triple negative & $0.0467 \pm 0.0019$ \\
\hline MDA-MB-231 & Triple negative & $0.0446 \pm 0.0009$ \\
\hline MDA-MB-468 & Triple negative & $0.0613 \pm 0.0017$ \\
\hline HCC1937 & Triple negative & $>5$ \\
\hline Hs578T & Triple negative & $>5$ \\
\hline
\end{tabular}

The $\mathrm{IC}_{50}$ values of KX-01 determined using an MTT assay as described in "Materials and Methods" are shown. ER, estrogen receptor; PR, progesterone receptor; HER2, human epidermal growth factor receptor type 2. 

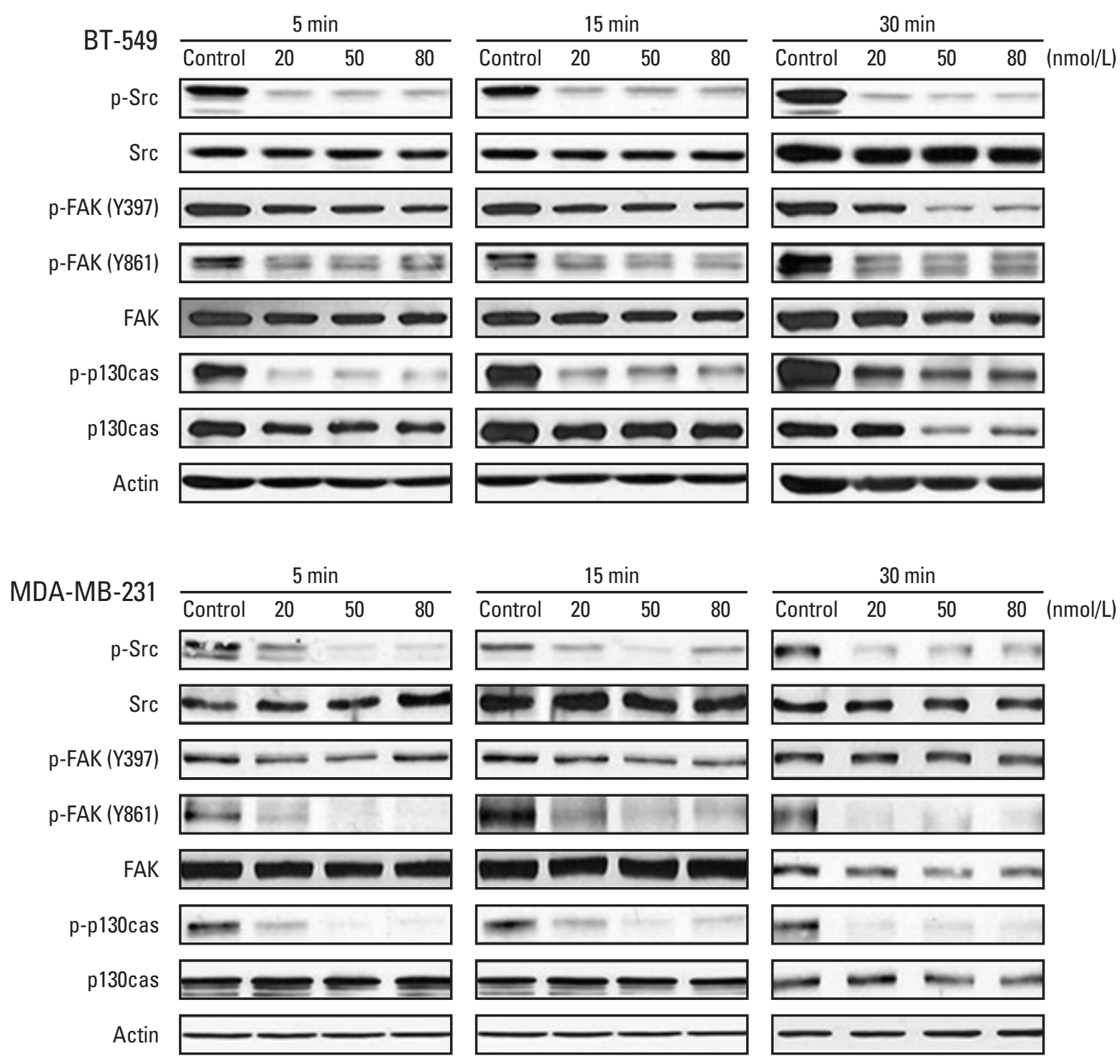

Fig. 1. KX-01 treatment in TNBC inhibits Src activity and the migration of cancer cells. (A) BT-549 and MDA-MB-231 cells were treated with KX-01 at the indicated time and dose. Western blot analysis showed molecular expression changes following KX-01 treatment. The active form of Src, FAK, and p130cas were all down-regulated by KX-01 treatment. (Continued to the next page)

3. KX-01 increases aneuploidy and induces mitotic catastrophe

KX-01 has microtubule polymerization inhibitor activity that leads to G2/M arrest. The substantial increase in the G2/M cell cycle population and expanded cell size with KX-01 treatment led us to consider the possibility of an increase in aneuploidy. Consequently, the DNA content was measured by flow cytometry analysis. First, we defined cells with DNA content of more than $6 \mathrm{~N}$ as the aneuploidy population. The aneuploidy population of each cell line was subsequently analyzed by flow cytometry. Impressively, KX-01 treatment caused induction of aneuploidy 10 to 18 times higher than each control (Fig. 3A). This escalation was only observed in KX-01-sensitive cell lines. Hs578T cell did not show any induction of the aneuploidy population. These data suggested that KX-01 induces defective mitosis through microtubule polymerization inhibition. 

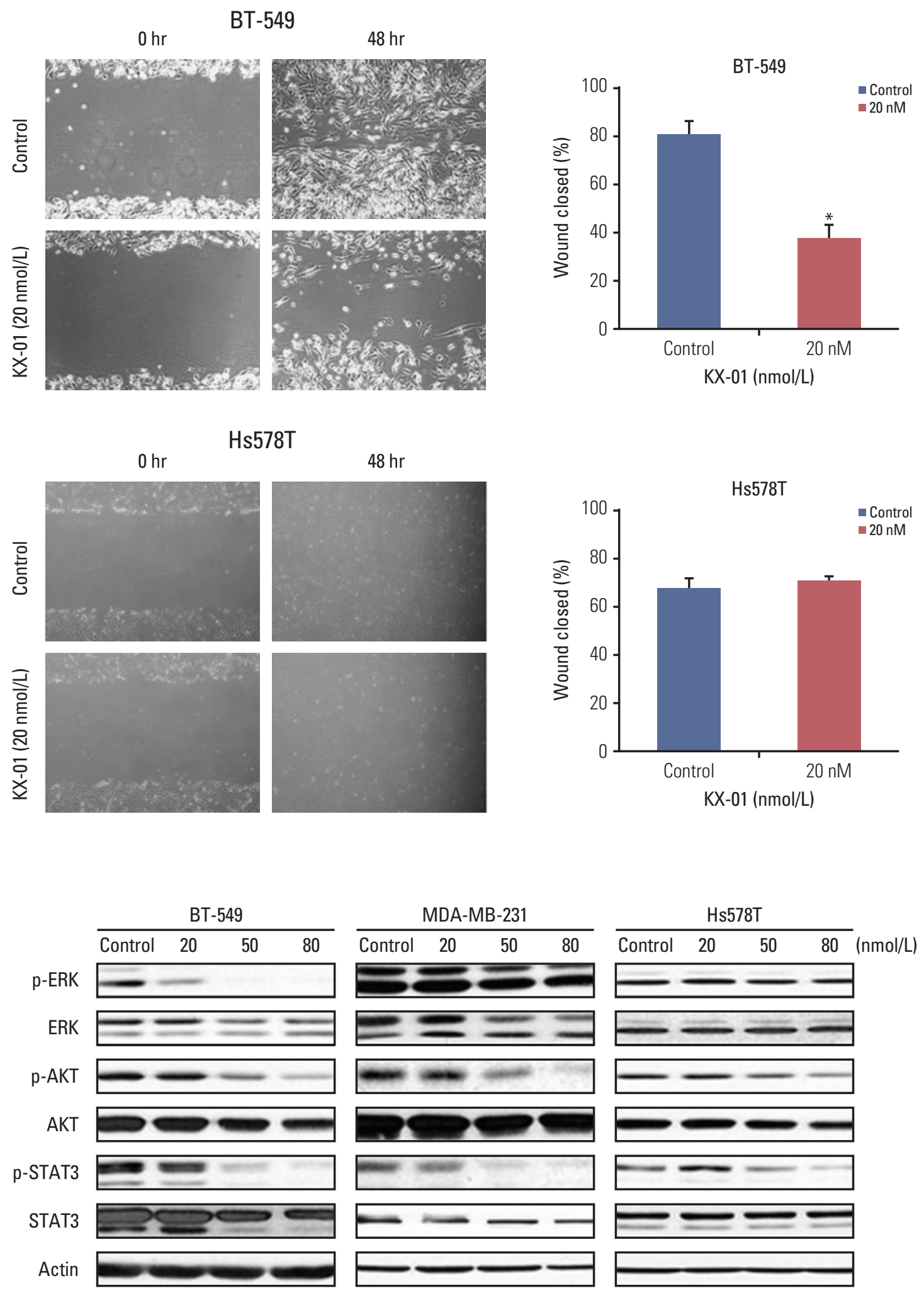

Fig. 1. (Continued from the previous page) (B) BT-549 and Hs578T cells were incubated with dimethyl sulfoxide (control) or KX-01 for 48 hours. Wound healing assay results demonstrate the migration inhibitory effect of KX-01. The columns are shown with error bars ( \pm standard error). * $\mathrm{p}<0.05$. (C) BT-549, MDA-MB-231, and Hs578T cells were exposed to KX-01 for 24 hours. Western blot results show molecular expression changes, which are related to Src signaling. 

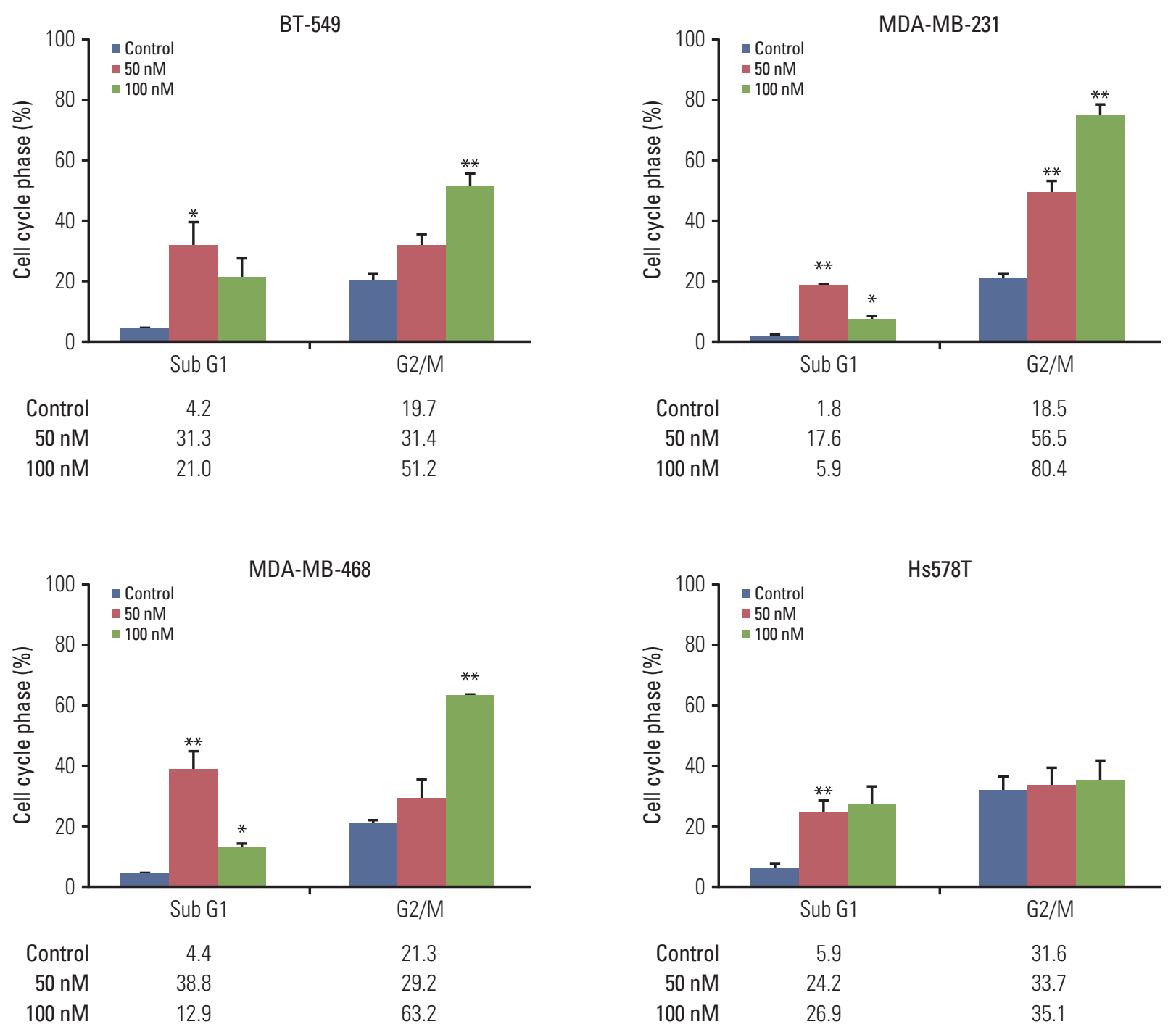

Fig. 2. KX-01 causes breast cancer cell death and G2/M cell cycle arrest. BT-549, MDA-MB-231, MDA-MB-468, and Hs578T cells were treated with the indicated concentrations of KX-01 for 48 hours. The percentages of cells in the G2/M or Sub G1 phase were determined by flow cytometry analysis. The columns represent the means of three independent experiments and are shown with error bars ( \pm standard error). ${ }^{*} \mathrm{p}<0.05,{ }^{* *} \mathrm{p}<0.005$.

Mitotic catastrophe, which is cell death during mitosis caused as a result of premature entry into the mitotic cycle or irregular mitosis, can be induced by microtubule inhibitors [12]. One of the representative morphologic changes of mitotic catastrophe is increased multinuclei or micronuclei. Thus, we examined whether KX-01 could induce these morphological changes. Through immunofluorescence assays, we confirmed an increased number of multi-nucleated cells in KX-01-sensitive MDA-MB-231 cells. In contrast, the less sensitive Hs578T cells did not show any changes (Fig. 3B). The number of multi-nucleated cells was analyzed, and significant increases in multi-nucleated cell populations were only observed among KX-01-sensitive cells (Fig. 3C). Similar results were obtained with other KX-01sensitive cell lines (S2 Fig.). Further investigations of microtubule arrangements were conducted to verify that the increase of multi-nucleated cells in KX-01-sensitive cell lines was caused by inhibition of microtubule polymerization by KX-01.

MDA-MB-231 cells were treated with KX-01 for 48 hours to identify any changes in microtubule polymerization. No significant changes were observed in cells treated with 


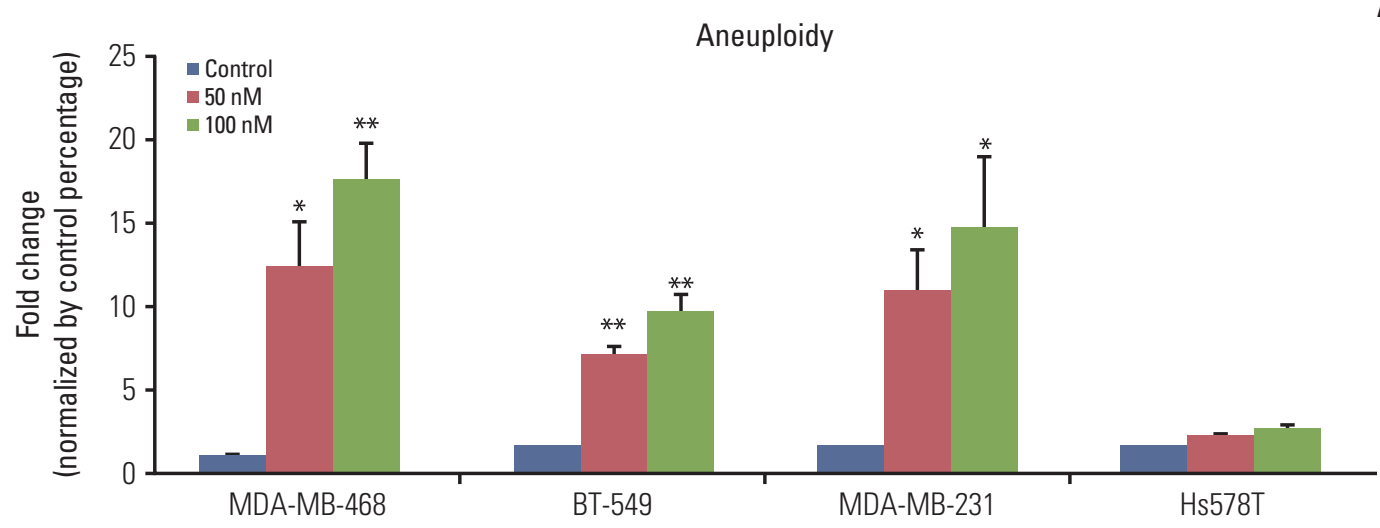

B

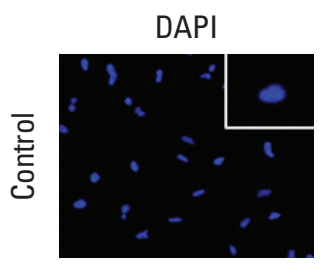

MDA-MB-231
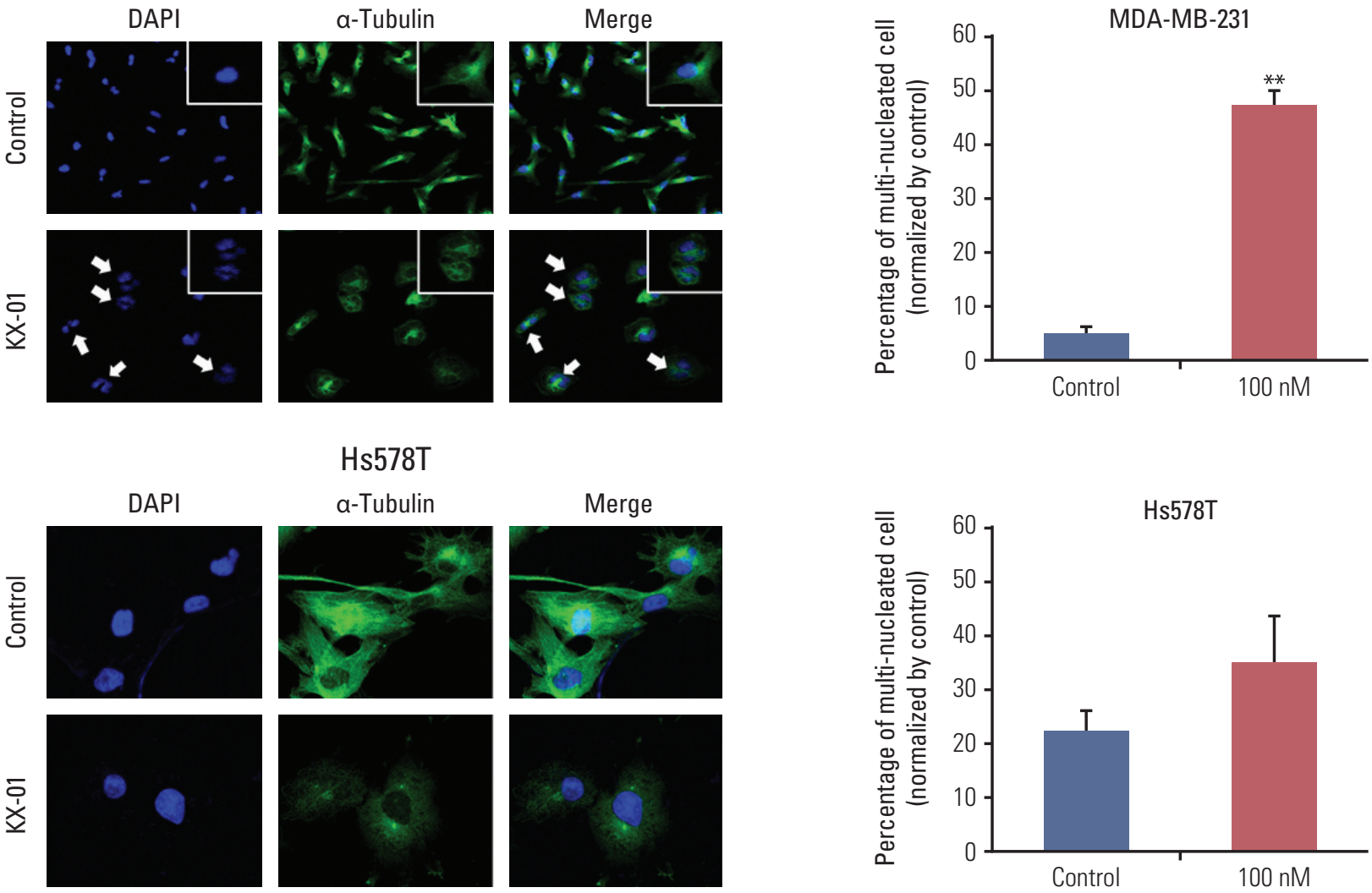

\section{Hs578T}

a-Tubulin
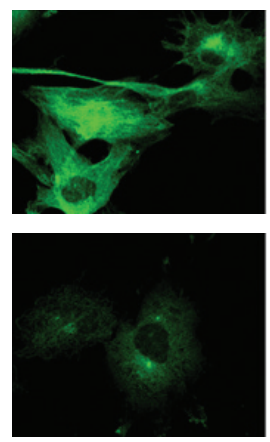
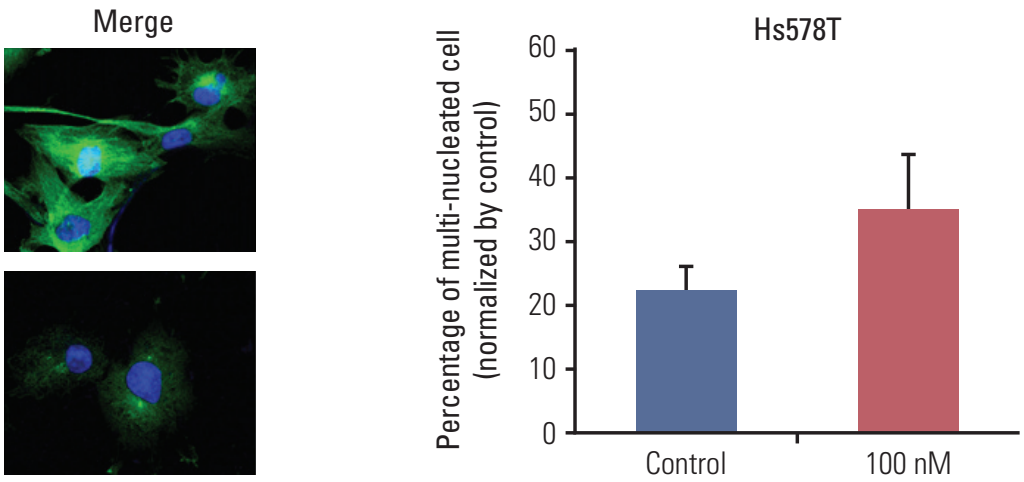

Fig. 3. KX-01 increases aneuploidy and induces mitotic catastrophe by inhibiting microtubule polymerization. (A) BT-549, MDA-MB-231, MDA-MB-468, and Hs578T cells were treated with indicated concentrations of KX-01 for 48 hours. The percentages of cells that contained more than $6 \mathrm{~N}$ were determined by flow cytometry analysis and compared to the control values. Each column is shown with error bars ( \pm standard error). ${ }^{*} \mathrm{p}<0.05,{ }^{* *} \mathrm{p}<0.005$. (B) MDA-MB-231 and Hs578T cells were incubated with $100 \mathrm{nmol} / \mathrm{L}$ of KX-01 or dimethyl sulfoxide (DMSO, control) for 24 hours. Confocal microscopy was used to observe the signal corresponding to $\alpha$-tubulin (green) and DNA was counterstained with DAPI (blue). Arrows indicate multinucleated cells. (C) One hundred cells in each KX-01 treatment level indicated were counted and the number of multinucleated cells were represented by a percentage. The columns represent the means of three independent experiments and are shown with error bars ( \pm standard error). ${ }^{* *} \mathrm{p}<0.005$. (Continued to the next page) 


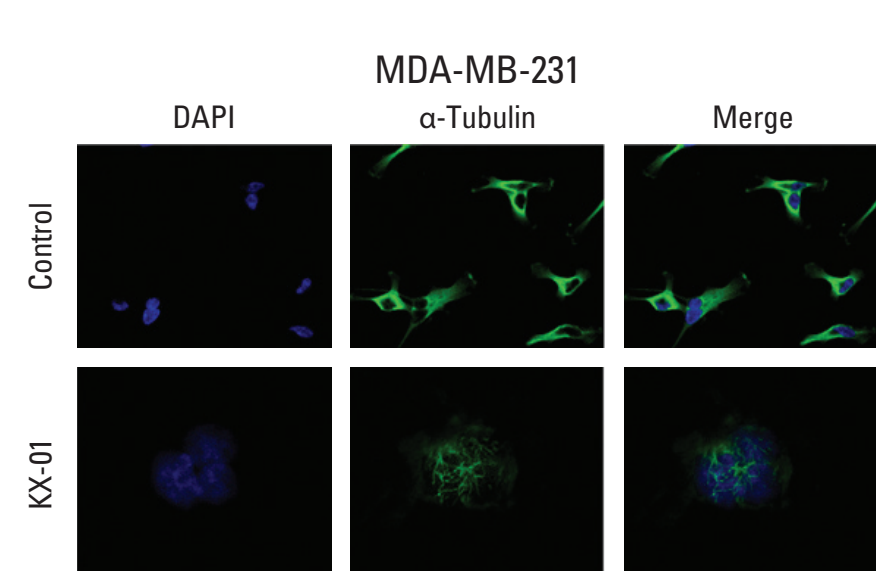

Fig. 3. (Continued from the previous page) (D) Microtubule conformation was analyzed with $100 \mathrm{nmol} / \mathrm{L}$ of KX-01 or DMSO control for 48 hours. Confocal microscopy was used to observe the signal corresponding to $\alpha$-tubulin (green) and DNA was counterstained with DAPI (blue).

DMSO control. However, in KX-01-treated cells, abnormal microtubule formations were observed and more cells were under the M phase (Fig. 3D). When observed in detail, only KX-01-sensitive cells failed to undergo cytokinesis due to KX-01 treatment (data not shown). Overall, these findings demonstrated that KX-01 induces mitotic catastrophe by inhibiting microtubule polymerization.

\section{KX-01 inhibits in vivo tumor growth in mice}

To confirm the antitumor effects of KX-01 observed in vitro, an in vivo mouse model was established using MDA-MB-231 cells. Briefly, 10 mice were divided into two groups and treated with vehicle or KX-01. After 4 weeks, the mice treated with KX-01 showed significantly delayed tumor growth (Fig. 4A). There were no significant weight changes in the mice treated with KX-01 (Fig. 4B). These results indicated that KX-01 had antitumor effects without obvious toxic effects on mice during the treatment period.

Tumor tissues from mice treated with KX-01 had lower levels of Ki-67 expression than the vehicle control tissues (Fig. 4C) [20,21], suggesting that KX-01 lowered the proliferation of the cancer cells. A TUNEL assay was used to measure the number of apoptotic cells. Tumor tissues from the KX-01 treatment group had significantly increased numbers of apoptotic cells relative to the vehicle control samples (Fig. 4C). Next, we determined whether trans-phosphorylation of Src was also inhibited by KX-01 treatment in vivo. Data from the Western blot assay demonstrated that phosphorFAK levels were reduced, as were p-Src levels (Fig. 4D). These in vivo data demonstrated the antitumor effects of KX-01 in the human TNBC MDA-MB-231 xenograft model.

\section{Discussion}

KX-01 is a small molecule that inhibits Src and tubulin polymerization. The effects of this compound are currently being investigated in phase II clinical trials $[15,16]$. In a previous study, KX-01 produced promising inhibitory effects in breast cancer cell lines [14]; however, the underlying mechanism of KX-01 antitumor activity was not fully demonstrated. In this study, we explored the action mechanism of the KX-01 antitumor activity in vitro in greater depth using TNBC cell lines. We demonstrated that KX-01 effectively inhibits TNBC cell growth and migration in a broad range of tumor cell lines. Moreover, this effect was, at least partially, due to down-regulation of Src signaling by KX-01 treatment. Previous studies demonstrated the down-regulation of p-paxillin, p-p130cas, and p-AKT without showing evidence of $\mathrm{p}$-Src down-regulation, which was the primary target for KX-01.

In the present study, we demonstrated the time course of inhibition of $\mathrm{p}$-Src levels by KX-01 treatment in vitro. Interestingly, we also showed that $\mathrm{Src}$ signaling-related molecules were still down-regulated, even after the p-Src activity was restored. The reason for the different time courses for these inhibitory responses may have clinical implications and will therefore be the subject of further investigations. Unlike other Src inhibitors, KX-01 is a reversible inhibitor that does not bind to the ATP pocket of Src [14,15,18]; consequently, it may have important unique biological and clinical effects. We confirmed that KX-01 can effectively inhibit Src signaling, reduce cell growth, and prevent cell migration. Despite the Src inhibitory effect of KX-01, basal expression of Src or 


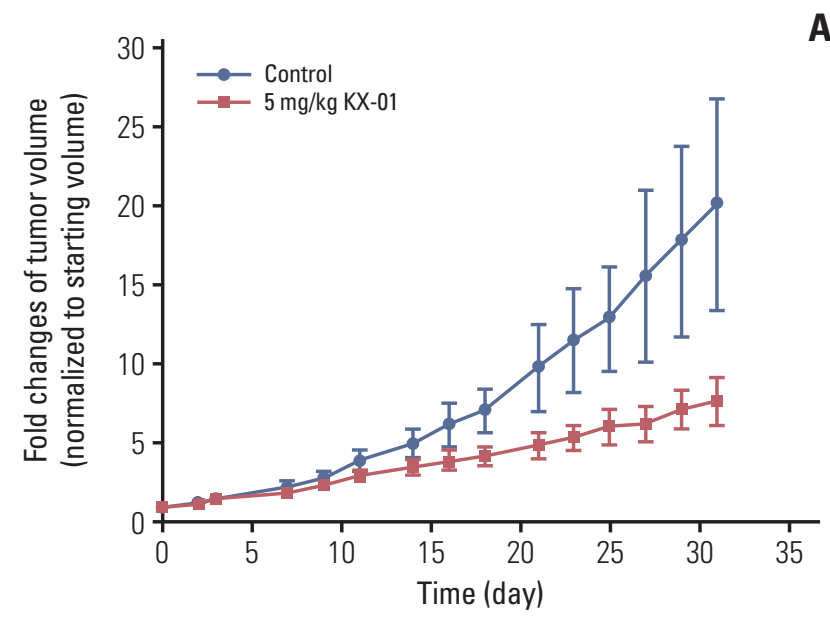

A

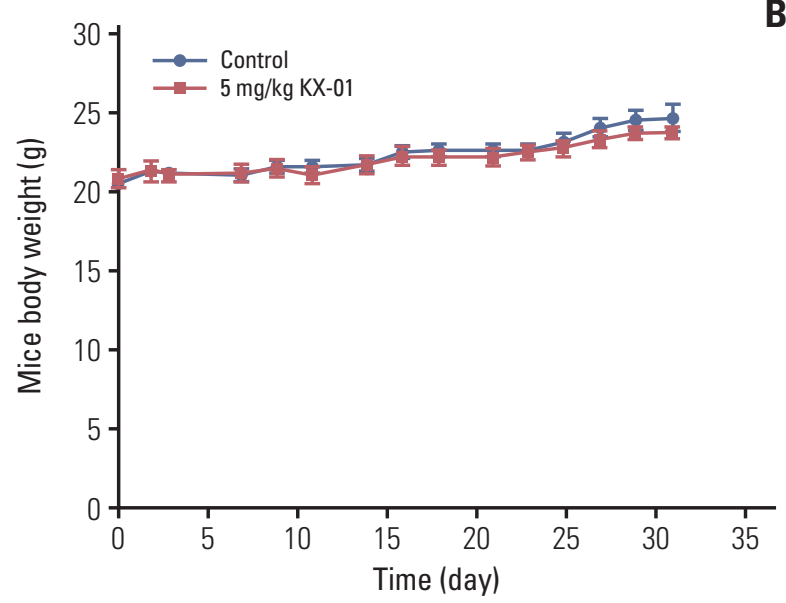

C

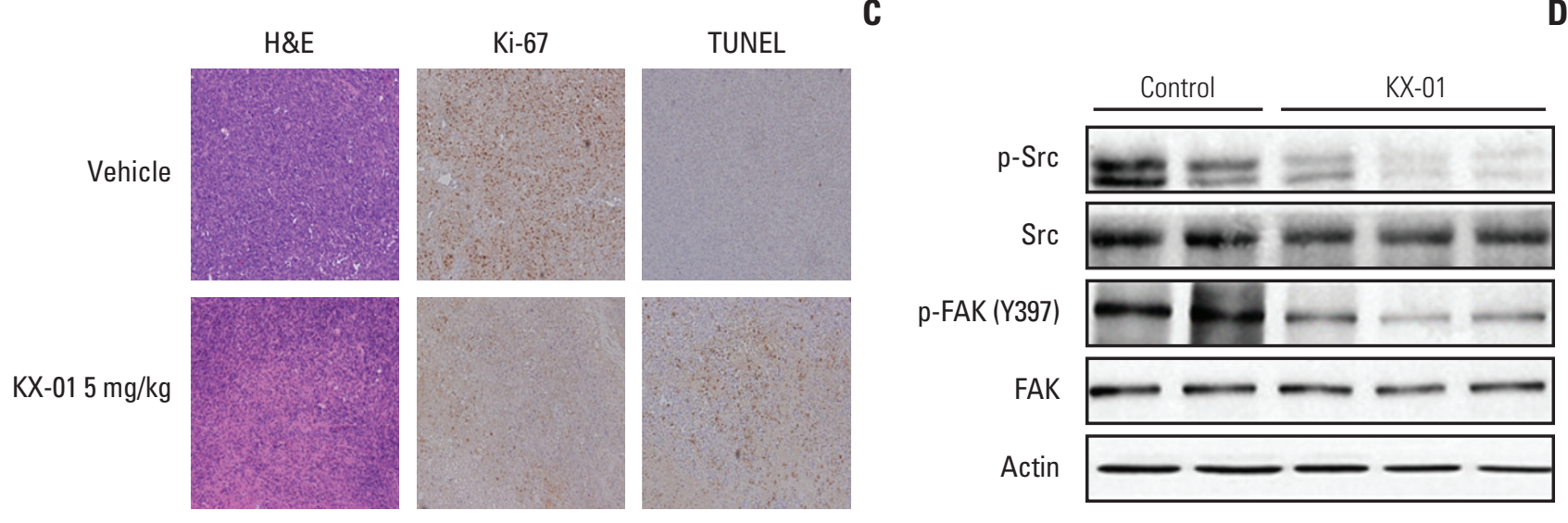

Fig. 4. KX-01 inhibits in vivo tumor growth in MDA-MB-231 mouse xenograft model. (A) BALB/c nude mice were injected with $5 \times 10^{7}$ MDA-MB-231 cells. The vehicle group received 10\% (2-hydroxypropyl)- $\beta$-cyclodextrin solution in phosphate buffered saline and the other group was treated with $5 \mathrm{mg} / \mathrm{kg}$ of KX-01 administered by oral gavage twice daily for 4 weeks. Tumor volumes were recorded as $\mathrm{mm}^{3}$ and compared to the starting tumor sizes values. (B) Mouse weights were measured three times weekly. Each dot indicates the mean mouse weight. No significant differences in body weight were detected. Mean values are shown \pm standard error. (C) The tumors were removed from the mice after KX-01 treatment ended, and pathologic examination was conducted using H\&E slides $(\times 200)$. Immunohistochemical staining for Ki-67 and terminal deoxynucletidyltransferase-mediated dUTP nick end labeling (TUNEL) assays showed decreased Ki-67 with increased apoptosis in KX-01 treatment tumors. (D) On the final day of treatment, total cell protein was extracted from mouse tissues for immunoblotting with the indicated antibodies.

p-Src levels was not associated with KX-01 sensitivity (S3 Fig.).

Another characteristic of KX-01 is the ability to inhibit microtubule polymerization $[14,15,18]$. This effect was observed when the KX-01 concentration exceeded 80 $\mathrm{nmol} / \mathrm{L}$, whereas Src and Src signaling inhibition was evident at concentrations as low as $20 \mathrm{nmol} / \mathrm{L}$. In the present study, we detected increased G2/M phase arrest and aneuploidy when the cells were treated with $100 \mathrm{nmol} / \mathrm{L}$ of KX-01. Significantly increased populations of multi-nucleated cells and aneuploid cells indicated that KX-01 induced mitotic catastrophe in vitro [9]. Our data also demonstrated abnormal microtubule polymerization induced by KX-01 treatment. Usually, microtubule targeting agents are limited 
in that normal cells may also be affected by the drug treatment. However, KX-01 does not appear to affect normal cells in an observable fashion. When MCF10A cells, a non-tumorigenic epithelial cell line, were treated with KX-01, G2/M cell phase arrest was not induced (data not shown). Thus, it seems that the antitumor effects of KX-01, inhibition of Src signaling and induction of mitotic catastrophe, are cancer specific phenomena.

Unlike sensitive cell lines (BT-549, MDA-MB-231, and MDA-MB-468), Hs578T cells were resistant to KX-01 treatment. Hs578T cells shows low levels of p-Src expression when compared with other sensitive cell lines (S3 Fig.). The expression of Src family members and additional downstream signaling does not appear to be affected by KX-01 treatment (Fig. 1C). Moreover, Hs578T cells tolerate microtubule aberrations by KX-01. Because Hs578T cells have hyper-tetraploid chromosome numbers, they appear to be less affected by deregulation of microtubule polymerization.

KX-01 showed the ability to overcome resistance induced by other anti-microtubule agents such as paclitaxel. In this study, KX-01 showed significant growth inhibitory activity in the paclitaxel resistant MCF7 cell line (S4 Fig.). Our data indicated that KX-01 could be an alternative to overcome resistance to paclitaxel or other anti-microtubule agents.

A relationship between Fyn (protein tyrosine kinase p59 $9^{\mathrm{Fyn}}$ ) and microtubule polymerization during neuronal cell development has been reported [22]. A previous study demonstrated that Fyn has the potential to modulate membrane associated $\gamma$-tubulin activities, which are important to initiating the formation of microtubules. Recruitment of tyrosinephosphorylated molecules during microtubule polymerization has also been described [23]. Recently, several studies demonstrated the possible involvement of Src and FAK in mitosis [24-27]. Therefore, we can hypothesize that Src inhibition activity could also contribute to the inhibitory effects on microtubule polymerization along with its direct binding and inhibitory effect of KX-01 on tubulin (data not yet published), although further investigation is needed to test this hypothesis.

Various inhibitors that target the ATP binding pocket of Src have been developed; however, the effects of these compounds in clinical trial have not been remarkable $[7,8]$. In clinical trials using dasatinib, which targets the ATP binding pocket of Src and other kinases, the compound did not show promising antitumor effects in solid tumor patients as a monotherapeutic agent $[6,28]$. Unlike previous Src inhibitors, KX-01 targets the non-ATP binding region. Therefore, this drug has less chance of blocking other tyrosine kinases that harbor the Src homology domain. A phase I clinical trial of KX-01 showed promising results against various solid tumors [17] and a phase Ib clinical trial is currently ongoing.

Here, we demonstrate the down-regulation of p-Src by
KX-01 in vitro for the first time. Previous studies only demonstrated p-Src inhibitory effects of KX-01 in vivo and indirectly showed Src inhibition by demonstrating inactivation of downstream molecules, such as p-paxillin or p-p130cas, instead of the inhibited Src itself. Thus, this paper is noteworthy because it directly shows that KX-01 regulates Src signaling via its direct inhibition of $\mathrm{p}$-Src. Moreover, we verified that treatment with $\mathrm{KX}-01$ aggravates the burden on the G2/M cell phase and causes abnormal mitosis, which induces mitotic catastrophe in TNBC cells. Src and microtubule dual inhibitory effects are the key characteristics that distinguish KX-01 from other Src inhibitors, and therefore make this drug a more promising treatment for TNBC.

The first-line chemoagent used for treatment of TNBC, paclitaxel, is also a well-known anti-microtubule drug; however, there are many paclitaxel-resistant patients. Based on its two inhibitory effects, it is anticipated that KX-01 has the potential to treat paclitaxel-resistant patients. Taken together, the results presented herein provide a better understanding of the action mechanism of KX-01, which may help future clinical trial design.

\section{Conclusion}

The results of this study demonstrate that KX-01 inhibits TNBC growth by modulating Src signaling and microtubule polymerization. Furthermore, we confirmed that KX-01 inhibits Src phosphorylation in vitro. Moreover, our findings verify that KX-01 treatment induces mitotic catastrophe. Overall, our efforts will broaden the understanding of the action mechanism of KX-01.

\section{Electronic Supplementary Material}

Supplementary materials are available at Cancer Research and Treatment website (http:// www.e-crt.org).

\section{Conflicts of Interest}

Y.J.B. has advised or consulted for and received research funding from Bayer, Novartis, Boeringer-Ingelheim, Roche/ Genentech, AstraZeneca, Merck Serano, MSD, Bristol-Myers Squibb, Eli Lilly, Pfizer, ONO, Taiho and GreenCross. S.A.I. 
is a recipient of research funds from AstraZeneca, Inc., and acts as a consultant and advisor for AstraZeneca, Roche, and Novartis. D.Y.O. is a recipient of research funds from AstraZeneca Inc. D.H. and J.Y.N.L. are employees of the Kinex Pharmaceuticals Corporation. The other authors declared that they have no conflicts of interest.

\section{Acknowledgments}

This research was partly supported by the Priority Research Centers Program and Basic Science Research Program through the National Research Foundation of Korea (NRF) funded by the Ministry of Education, Science and Technology (2009-0093820) and the Basic Science Research Program through the NRF funded by the Ministry of Science, ICT and Future Planning (2015R1A2A2A01004655). We also sincerely appreciate Mrs. Myung-Hwa Lee and Mr. Hyuk Jin Chung for their dedication to cancer research funding, even though Mrs. Lee was suffering from metastatic breast cancer.

\section{References}

1. Cleator S, Heller W, Coombes RC. Triple-negative breast cancer: therapeutic options. Lancet Oncol. 2007;8:235-44.

2. Tryfonopoulos D, Walsh S, Collins DM, Flanagan L, Quinn C, Corkery B, et al. Src: a potential target for the treatment of triple-negative breast cancer. Ann Oncol. 2011;22:2234-40.

3. Hiscox S, Nicholson RI. Src inhibitors in breast cancer therapy. Expert Opin Ther Targets. 2008;12:757-67.

4. Finn RS. Targeting Src in breast cancer. Ann Oncol. 2008;19: 1379-86.

5. Chen T, Pengetnze Y, Taylor CC. Src inhibition enhances paclitaxel cytotoxicity in ovarian cancer cells by caspase9-independent activation of caspase-3. Mol Cancer Ther. 2005;4:217-24.

6. Finn RS, Bengala C, Ibrahim N, Roche H, Sparano J, Strauss LC, et al. Dasatinib as a single agent in triple-negative breast cancer: results of an open-label phase 2 study. Clin Cancer Res. 2011;17:6905-13.

7. Gucalp A, Sparano JA, Caravelli J, Santamauro J, Patil S, Abbruzzi A, et al. Phase II trial of saracatinib (AZD0530), an oral SRC-inhibitor for the treatment of patients with hormone receptor-negative metastatic breast cancer. Clin Breast Cancer. 2011;11:306-11.

8. Carey L, Winer E, Viale G, Cameron D, Gianni L. Triple-negative breast cancer: disease entity or title of convenience? Nat Rev Clin Oncol. 2010;7:683-92.

9. Castedo M, Perfettini JL, Roumier T, Andreau K, Medema R, Kroemer G. Cell death by mitotic catastrophe: a molecular definition. Oncogene. 2004;23:2825-37.

10. Gardner MK, Zanic M, Howard J. Microtubule catastrophe and rescue. Curr Opin Cell Biol. 2013;25:14-22.

11. Topham CH, Taylor SS. Mitosis and apoptosis: how is the balance set? Curr Opin Cell Biol. 2013;25:780-5.

12. Vakifahmetoglu H, Olsson M, Zhivotovsky B. Death through a tragedy: mitotic catastrophe. Cell Death Differ. 2008;15: 1153-62.

13. Vitale I, Galluzzi L, Castedo M, Kroemer G. Mitotic catastro- phe: a mechanism for avoiding genomic instability. Nat Rev Mol Cell Biol. 2011;12:385-92.

14. Anbalagan M, Ali A, Jones RK, Marsden CG, Sheng M, Carrier $\mathrm{L}$, et al. Peptidomimetic Src/pretubulin inhibitor KX-01 alone and in combination with paclitaxel suppresses growth, metastasis in human ER/ PR/HER2-negative tumor xenografts. Mol Cancer Ther. 2012;11:1936-47.

15. Anbalagan M, Carrier L, Glodowski S, Hangauer D, Shan B, Rowan BG. KX-01, a novel Src kinase inhibitor directed toward the peptide substrate site, synergizes with tamoxifen in estrogen receptor alpha positive breast cancer. Breast Cancer Res Treat. 2012;132:391-409.

16. Antonarakis ES, Heath EI, Posadas EM, Yu EY, Harrison MR, Bruce JY, et al. A phase 2 study of KX2-391, an oral inhibitor of Src kinase and tubulin polymerization, in men with bonemetastatic castration-resistant prostate cancer. Cancer Chemother Pharmacol. 2013;71:883-92.

17. Naing A, Cohen R, Dy GK, Hong DS, Dyster L, Hangauer DG, et al. A phase I trial of KX2-391, a novel non-ATP competitive substrate-pocket- directed SRC inhibitor, in patients with advanced malignancies. Invest New Drugs. 2013;31:967-73.

18. Liu T, Hu W, Dalton HJ, Choi HJ, Huang J, Kang Y, et al. Targeting SRC and tubulin in mucinous ovarian carcinoma. Clin Cancer Res. 2013;19:6532-43.

19. Kang S, Min A, Im SA, Song SH, Kim SG, Kim HA, et al. TGF$\beta$ suppresses COX-2 expression by tristetraprolin-mediated RNA destabilization in A549 human lung cancer cells. Cancer Res Treat. 2015;47:101-9.

20. Min A, Im SA, Yoon YK, Song SH, Nam HJ, Hur HS, et al. RAD51C-deficient cancer cells are highly sensitive to the PARP inhibitor olaparib. Mol Cancer Ther. 2013;12:865-77.

21. Nam HJ, Im SA, Oh DY, Elvin P, Kim HP, Yoon YK, et al. Antitumor activity of saracatinib (AZD0530), a c-Src/Abl kinase inhibitor, alone or in combination with chemotherapeutic agents in gastric cancer. Mol Cancer Ther. 2013;12:16-26.

22. Macurek L, Draberova E, Richterova V, Sulimenko V, Suli- 
menko T, Draberova L, et al. Regulation of microtubule nucleation from membranes by complexes of membrane-bound gamma-tubulin with Fyn kinase and phosphoinositide 3-kinase. Biochem J. 2008;416:421-30.

23. Kasahara K, Nakayama Y, Nakazato Y, Ikeda K, Kuga T, Yamaguchi N. Src signaling regulates completion of abscission in cytokinesis through ERK/MAPK activation at the midbody. J Biol Chem. 2007;282:5327-39.

24. Nakayama Y, Matsui Y, Takeda Y, Okamoto M, Abe K, Fukumoto Y, et al. c-Src but not Fyn promotes proper spindle orientation in early prometaphase. J Biol Chem. 2012;287: 24905-15.

25. Soeda S, Nakayama Y, Honda T, Aoki A, Tamura N, Abe K, et al. v-Src causes delocalization of Mklp1, Aurora B, and
INCENP from the spindle midzone during cytokinesis failure. Exp Cell Res. 2013;319:1382-97.

26. Park AY, Shen TL, Chien S, Guan JL. Role of focal adhesion kinase Ser-732 phosphorylation in centrosome function during mitosis. J Biol Chem. 2009;284:9418-25.

27. Rea K, Sensi M, Anichini A, Canevari S, Tomassetti A. EGFR/ MEK/ERK/CDK5-dependent integrin-independent FAK phosphorylated on serine 732 contributes to microtubule depolymerization and mitosis in tumor cells. Cell Death Dis. 2013;4:e815.

28. Chee CE, Krishnamurthi S, Nock CJ, Meropol NJ, Gibbons J, Fu P, et al. Phase II study of dasatinib (BMS-354825) in patients with metastatic adenocarcinoma of the pancreas. Oncologist. 2013;18:1091-2. 